REVIEW

\title{
Sex differences in the social brain and in social cognition
}

Edited by Sara Bulgheroni and Junie Warrington.

Alice Mado Proverbio ${ }^{1}$ (D)

${ }^{1}$ Milan Center for Neuroscience| Department of Psychology, University of Milano-Bicocca, Milan, Italy

Correspondence

Alice Mado Proverbio, Department of Psychology, University of Milano-Bicocca, Piazza dell'Ateneo Nuovo 1, 20126, Milan, Italy.

Email: mado.proverbio@unimib.it

Funding Information

Università degli Studi di Milano-Bicocca

\section{Abstract}

Many studies have reported sex differences in empathy and social skills. In this review, several lines of empirical evidences about sex differences in functions and anatomy of social brain are discussed. The most relevant differences involve face processing, facial expression recognition, response to baby schema, the ability to see faces in things, the processing of social interactions, the response to the others' pain, interest in social information, processing of gestures and actions, biological motion, erotic, and affective stimuli. Sex differences in oxytocin-based parental response are also reported. In conclusion, the female and male brains show several neuro-functional differences in various aspects of social cognition, and especially in emotional coding, face processing, and response to baby schema. An interpretation of this sexual dimorphism is provided in the view of evolutionary psychobiology.

\section{Keywords}

baby schema | empathy | facial expression | gender | sex differences

\section{Significance}

This review is particularly relevant because it focuses on an extensive neuroimaging and electrophysiological literature on functional and anatomical sex differences in social cognition, thus revealing new and hitherto unknown knowledge.

\section{INTRODUCTION}

Genetic and hormonal influences are long known to affect the human brain and determine a variety of anatomical and functional differences between the two sexes. These sex differences would concern: overall brain volume (Zaidi, 2010), neuronal density and white matter thickness (Luders \& Toga, 2010), microstructure of the thalamus, corpus callosum, and cingulum (Menzler et al., 2011), structure/size of anterior hypothalamus, of the bed nucleus of the stria terminalis, of the posterodorsal region of the medial amygdala, and of the preoptic area (see Cahill, 2006; 
Hines, 2020) for comprehensive reviews). The cerebral sexual dimorphism would support marked diversities in reproductive, parental, and social behavior. In addition, prenatal and postnatal differences in testosterone concentration (between females and males) would heavily affect the brain physiology, also modulating dendritic growth, spine density, density of brain receptors, neurogenesis, and gliogenesis (McCarthy et al., 2012). A rapidly burgeoning literature now documents significant sex differences in the reactivity to/efficacy of drugs and pharmaceutical molecules, as well as in the incidence of neurodegenerative, neurological, and psychiatric diseases (see the entire volume dedicated to sex differences in the brain and edited by Cahill, 2017).

Besides anatomical and physiological diversities, some functional and mental differences between men and women have long been identified (e.g., Kimura, 1980) and more recently supported by neuroimaging studies (e.g., verbal fluency Sokołowski et al., 2020) and empathy (BaronCohen, 2002) in favor of female gender and spatial rotation ability (Voyer et al., 1995) in favor of the male gender. Lately an increasingly large amount of evidence has accumulated indicating a sex difference in social and affective processes (e.g., Anderson et al., 2013; McDuff et al., 2017; Perrett et al., 1998; Stevens \& Hamann, 2012; Thompson \& Voyer, 2014). In this review, we will examine a large corpus of behavioral, EEG/ERPs, and neuroimaging evidence in the recent literature supporting the existence of sex differences in the social brain.

The main known sex differences in social cognition refer to: hemispheric lateralization for face processing (Bourne, 2005; Proverbio, De Gabriele, et al., 2011; Proverbio et al., 2006, 2010, 2012), facial expression encoding (e.g., Connolly et al., 2019; Orozco \& Ehlers, 1998; Sawada et al., 2014; Wingenbach et al., 2018), face gender encoding (Proverbio et al., 2010a), interest in social scenes and human faces (Pavlova et al., 2014, 2015; Proverbio, 2017; Proverbio et al., 2008), emotional response to negative affective information (Hofer et al., 2007; Klein et al., 2003), empathy for pain (Han et al., 2008; Proverbio et al., 2009; Singer et al., 2004), understanding gestures, body language, and intentions (Canessa et al., 2012; Proverbio, De Gabriele, et al., 2011), parental response to baby schema (Glocker, Langleben, Ruparel, Loughead, Gur, et al., 2009; Glocker, Langleben, Ruparel, Loughead, Valdez, et al., 2009; Sander et al., 2007; Seifritz et al., 2003).

\subsection{Hemispheric asymmetries for face processing}

While the classical neuroimaging literature reports a right hemispheric dominance for face processing in humans (Kanwisher et al., 1997; Rossion et al., 2003; Sergent et al., 1992), regardless of gender of viewers, new recent data have shown that face processing is less lateralized in females than males (Kiesow et al., 2020; Liu et al., 2020; Proverbio et al., 2010b; Proverbio \& Galli, 2016). Others authors suggested that facial processing involved both the right and left hemispheres (Haxby et al., 1999) but that they would contribute differently in response to familiar or unfamiliar faces. Overall, the literature is very inconsistent about hemispheric lateralization of facial processing since virtually no neuroimaging study has ever considered viewer's sex in the past. At this regard, it is of great interest to note that face-specific N170 responses of event-related potentials (ERPs) are found to be bilateral or even left-sided in studies in which female participants are the majority (e.g., Jemel et al., 2005).

Two ERP studies (Proverbio et al., 2006b, 2012) have shown a face-specific activation of the left occipito-temporal area, namely of the fusiform face area (FFA), in females during processing of facial stimuli (as reflected by N170 component of ERPs) and of the right homologous FFA, in males. More in detail, Proverbio and coworkers (2012) recorded ERPs to 400 stimuli (faces of adults, children, and infants, tools) in 50 participants, in a landscape detection task. The results showed a lack of difference in the amplitude of N170 to objects in the two sexes. However, it was found that N170 to faces was of greater amplitude over the right in males, and bilateral in females. 
Furthermore, the left face area in males was not responding to people's age (it did not discriminate between adult, children, or baby faces) but only the right one, while the age discriminative response was bilateral in females.

Overall, these findings fit with previous data providing evidence of a sex difference in the hemispheric lateralization of perceptual and cognitive processes. For example, several studies have shown more strongly lateralized functions in males versus females, including language and visuospatial processes. In particular, females would rely more on interhemispheric communication during tasks involving 3D mental rotation (Kurth et al., 2018), and would show a more bilateral activation of brain areas devoted to linguistic functions (Hausmann, 2016; Kansaku \& Kitazawa, 2001; Shaywitz et al., 1995). Bourne (2005) investigated the existence of hemispheric asymmetries during face processing. The task consisted of viewing chimeric faces with different facial expressions and determining which one looked more positive. Again, the data provided evidence of a stronger hemispheric asymmetry in men compared to women, with a perceptual advantage of the left visual field (right hemisphere) in the latter group. Tiedt et al. (2013) reported similar findings in an ERP study on the interhemispheric transfer-time (IHTT) of facial information estimated through N170 latency. They showed a sex difference in IHTT with earlier N170 latencies in the right-to-left direction $(170 \mathrm{~ms})$ as opposed to the left-to-right direction $(185 \mathrm{~ms})$, while IHTT was symmetric in women. Quite similar findings were also reported by Proverbio et al. (2012) who concluded that asymmetry in callosal transfer times in males might be ascribed to quicker transfer times of facial inputs through pathways departing from the more efficient (right) to the less efficient (left) hemisphere. Likewise, the lack of asymmetry in females might suggest that both face areas are equally specialized.

\subsection{Affective facial expressions and emotions}

Empirical evidence of females' greater accuracy in interpreting facial mimicry has also been provided (Connolly et al., 2019; Sawada et al., 2014; Thompson \& Voyer, 2014; Williams et al., 2009; Wingenbach et al., 2018). This finding is typically interpreted in an evolutionary perspective, considering the primary role of mothers in the early interactions with infants. In this regard, Proverbio and coworkers (2007) examined the role of biological sex and expertise in understanding infant expressions in a large group of men and women who differed in their experience with babies. Women showed a better performance and decoding accuracy than men; furthermore, familiarity with babies (linked to profession) improved decoding of facial expressions but only in women. These results suggest an interaction between expertise and a biologically driven female inclination to better comprehend babies' facial expressions (i.e., an interaction between sex and cultural factors).

In an electrophysiological study performed on the same set of stimuli (Proverbio et al., 2006b), it was investigated whether viewers' sex affected the perceptual response at different stages of visual processing during a decision task of infant joy/pain expressions. All infants were unknown to participants. Overall, the occipital P1 response was earlier and much greater in female than male participants. Furthermore, P1 latency was faster in response to pain than neutral expressions only in women, thus showing a prioritized processing of biologically relevant information in the female brain (Figure 1). 


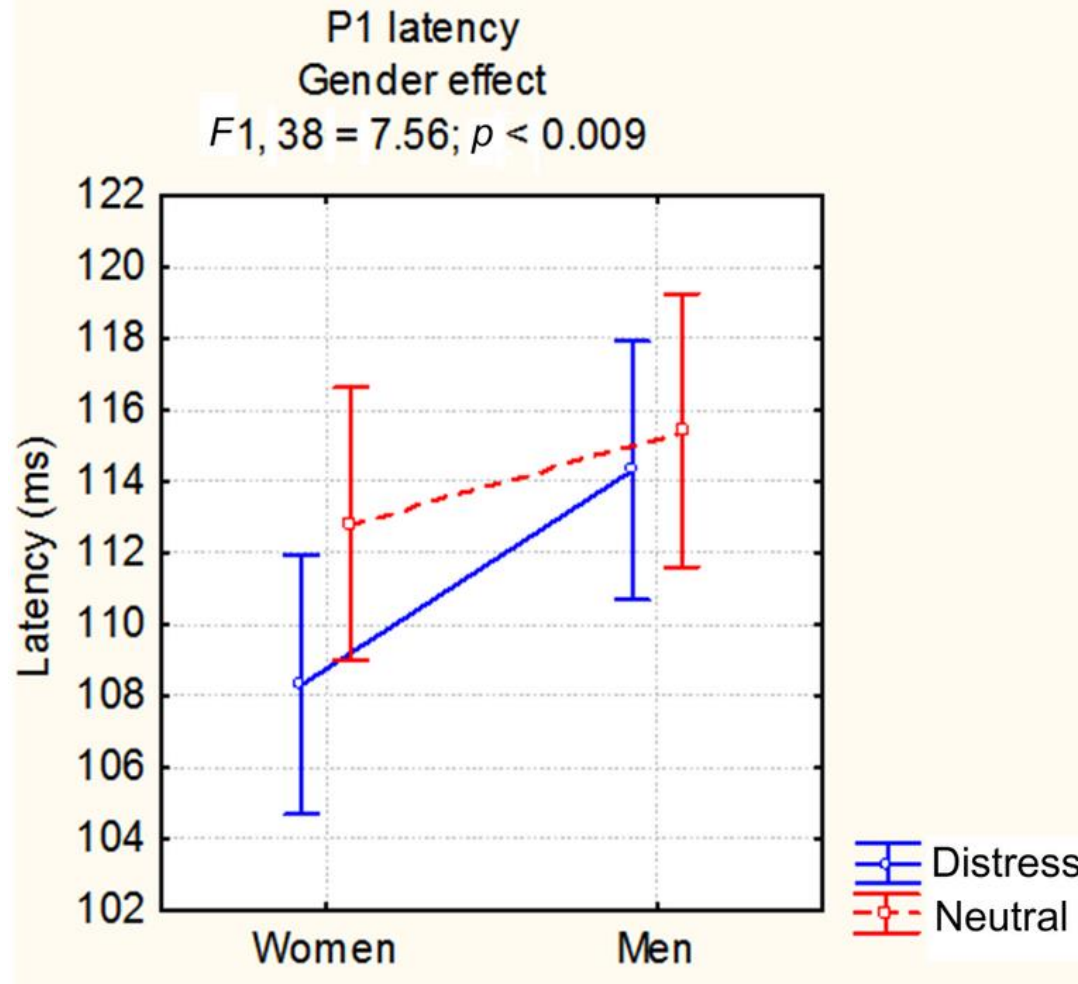

P1 latency

Gender effect

$F 1,38=7.56 ; p<0.009$

FIGURE 1Mean latency of P1 component recorded at lateral occipital area (independent of hemispheric site) and analyzed according to viewer's sex and type of facial expression (taken and modified from Proverbio et al., 2006b study, protected by CC BY 2.0)

The role of sex in the emotion comprehension and psychological reaction to emotional visual stimuli has been also investigated (Proverbio, 2017; Stevens \& Hamann, 2012). Quite recently Li et al. (2020) analyzed a large data set of 970 subjects (508 women) engaged in identifying negative facial emotions and neutral shape targets finding that women were more sensitive to fearful stimuli, whereas men were more sensitive to anger-provoking stimuli.

In general, stimuli producing a larger arousal response would undergo a prioritized processing because of their biological relevance, for example, baby faces would stimulate an instinctual parental response, especially in women. In contrast, erotic stimuli (such as nudity) would be particularly arousing for males versus females. Sabatinelli et al. (2004) showed that perceiving erotic images induced a much larger activation of associative visual areas in males than women. Indeed, Huynh and coworkers (2012) reported that high-intensity (vs. low-intensity and neutral) erotic movies deactivated the primary visual area in women. Conversely, perception of scenes stimulating an empathic response (such as, body mutilations) triggered a greater activity in the extra-striate visual area of females versus males. These findings seem to indicate that brain arousal and attentional allocation devoted to social stimulus depends on their specific biological relevance for the viewers.

\subsection{Parental response}

A wide literature shows that the visual and the orbitofrontal cortices of adult viewers are particularly aroused by the view of baby faces (Nitschke et al., 2004). Viewing an infant would provide a positive feeling (e.g., tenderness or cuteness) especially in women, but also in men, via the dopaminergic reward system. This effect is known as "baby schema" effect, and besides the 
enhanced affective valance it results in a deeper processing and attentional orienting toward children versus adult faces (Brosch et al., 2007; Glocker, Langleben, Ruparel, Loughead, Gur, et al., 2009; Luo et al., 2011; Proverbio, De Gabriele, et al., 2011; Proverbio, Riva, Zani, et al., 2011; see also the review by Hahn \& Perrett, 2014). Other behavioral studies showed how women might be more responsive to baby schema than men and more skilled in comprehending infant expressivity (Babchuk et al., 1985; Proverbio et al., 2007). For example, in an EEG/ERP study (Proverbio et al., 2006) aimed at investigating the neural response to baby schema in female and male adult people, data showed the sensory response to faces (P1) was of greater amplitude in women than men. This might suggest a greater perceptual sensitivity or attentive response in females versus males at the view of unrelated infants. Other studies showed that baby faces were more perceptually salient for women than men (Hahn et al., 2013; Parsons et al., 2011, 2013). In addition, infant faces would capture females' attention to a greater extent than adult faces; conversely, infant faces would capture male's attention much less than faces of the opposite sex, that is, female faces (Cárdenas et al., 2013).

Several interesting neuroimaging studies (Glocker, Langleben, Ruparel, Loughead, Valdez, et al., 2009; Kringelbach et al., 2008; Leibenluft et al., 2004) have investigated which neural systems are involved in the "parental response" to infants, and identified a neural network of structures based primarily on the orbitofrontal cortex. For example, Leibenluft and coauthors (2004) recorded the brain activation of mothers while viewing photographs of their own infants. They wished to get some knowledge on the neural correlates of "maternal love." It was found a focus of activity in the orbitofrontal area and in related regions belonging to the pleasure/reward system, including the periaqueductal gray matter.

In an electrophysiological study (Peltola et al., 2014), it was tested the associations between motherhood and genetic variations in oxytocin receptors, and bioelectrical and behavioral responses to facial expressions of babies and adults. Both mothers (as opposed to nulliparous females) and women carrying the rs $53576 \mathrm{GG}$ variant of $O X T R$ gene showed larger discriminative N170 responses to strong versus mild negative facial expressions of infants. This result suggests that the visual cortex responsivity to baby schema depends both on genetic (individual differences) and biological factors (being a mother or not). Overall, being a parent has been associated with a greater sensitivity to infant facial expressions. This has been demonstrated in an electrophysiological study performed in parent versus nulliparous adults where it was found that perceptual N160 response reflected the earliest discrimination of mild versus strong painful facial expressions in parents (especially in mothers) but not in nulliparous individuals, thus suggesting again a strong interaction of genetic predisposition and parental status (Proverbio et al., 2006a). Again, the data showed larger P3 responses in mothers versus all other groups (i.e., males and nulliparous females), possibly indicating a greater perceptual sensitivity (or enhanced arousal) in mothers, at the view of unknown babies.

As far as for auditory social information are concerned, the neuroscientific literature reported larger brain responses in women than men while listening to infant vocalizations such as cry and laughter (e.g., Sander et al., 2007; Seifritz et al., 2003). These data also seem to suggest the hypothesis of a sex difference in the parental response to infantile nonverbal signals. It is conceivable that many of the sex differences in social cognition might be related to the genetically determined role of human females as primary caregivers of the offspring (instead of fighters/hunters, as suggested by Kuhn \& Stiner, 2006). For example, the robust evidence linking testosterone exposure to sex-typed play (e.g., playing or not with dolls) suggest how behavior is strongly modulated by sexual genes (Hines, 2020; Leveroni \& Berenbaum, 1998), but it cannot be rule out the role of socialization and upbringing style. 


\subsection{Interest in social stimuli}

Some electrophysiological literature provided evidence of a greater female electrocortical response to social information (e.g., faces or people) as opposed to inanimate stimuli. In a study by Proverbio et al. (2008), participants viewed hundreds of pictures depicting real persons versus sceneries (urban or natural landscapes) while their EEG signals were recorded. The results showed larger amplitude $\mathrm{N} 2$ responses in women, but not in men, in response to social than inanimate information. Source reconstruction of brain signals evidenced a bilateral activation of face-related regions in response to persons. In addition, sex differences were found with the unique activation of the superior temporal gyrus and the right extra-striate body area (EBA) in women, and of the left para-hippocampal area in men. This was interpreted as female greater interest in (or attention to) social signals (i.e., bodies and faces).

This interest for social information might draw more attention to visual stimuli. Indeed, a considerable literature has accumulated showing that females are more accurate than males in decoding facial mimicry. Many studies have reported sex differences in the ability to perceive, process, express, and experience emotions. Overall, females seem better skilled in the analysis and comprehension of social signals, and more interested in conspecifics. Again, female children typically spend more time with infants, younger siblings, and dolls than male children. While it is problematic to determine whether this socially oriented behavior is due to cultural factors (e.g., society models, upbringing style) or to genes, comparative studies on monkeys seem to point to the second direction.

\subsection{Action and body language understanding}

Several sex differences have been found in the way female and male brain processes actions and gestures. Proverbio et al. (2010)'s study, for example, reported earlier and greater ERP responses to incongruent actions in women than men. Perception of congruent actions (e.g., a woman looking attentively at road map) was compared with that of incongruent actions (e.g., a man in a kitchen cutting a carrot with an axe). Data showed an earlier discrimination between congruent and incongruent actions in women, indexed by a parietal N200 to response. The inverse solution applied to this component located the neural sources of this effect in the inferior parietal and left inferior frontal cortex (among other areas), corresponding to the mirror neuron network. In addition, the later anterior N400 component (greater to incongruent behavior) was found enhanced in women versus men (see Figure 2). The N400 source analysis showed slightly different neural circuits in two sexes, with an activation of the limbic system and cingulate cortex in women, and of the ventromedial prefrontal cortex in men, which has been interpreted as a more rational than affective reaction in men than women. 


\section{Congruent}
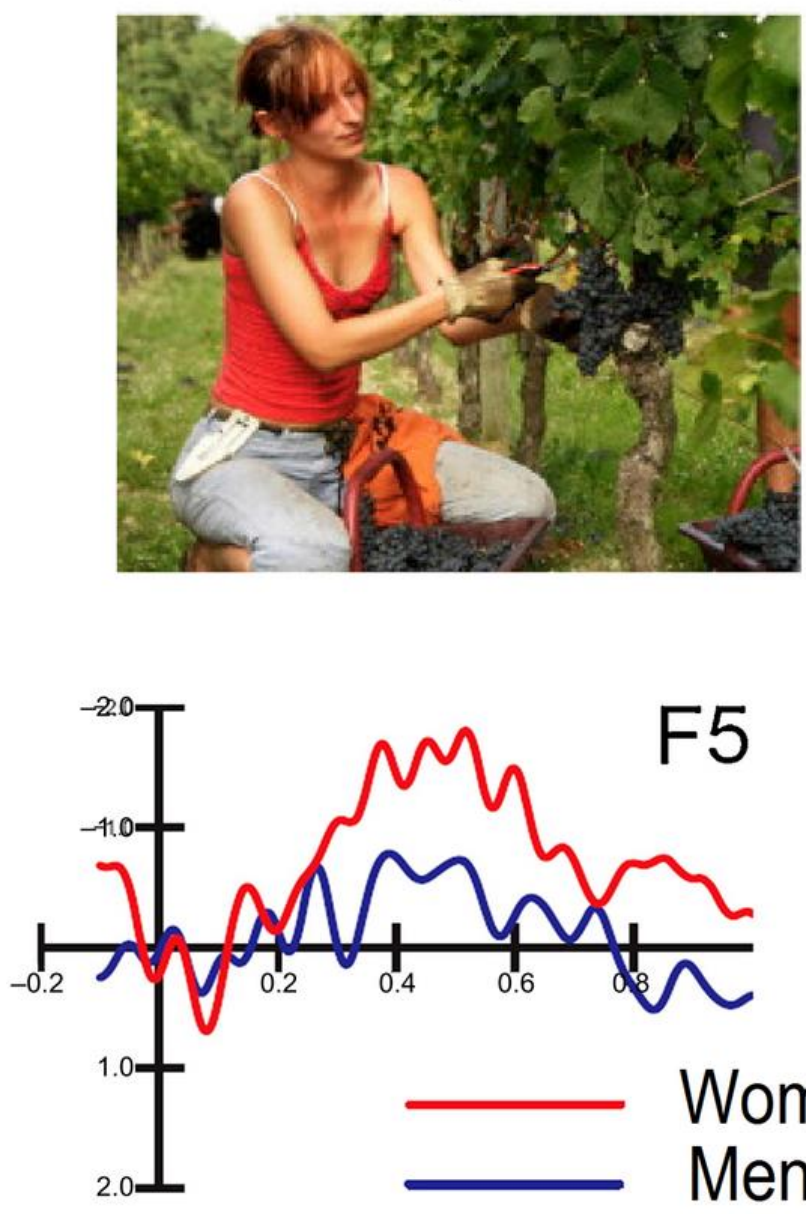

\section{Incongruent}

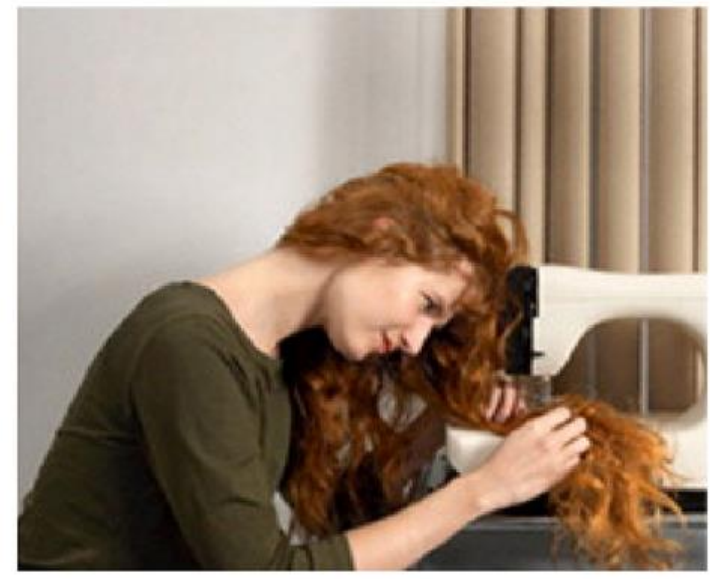

$\mathrm{N} 400$

Women

Men

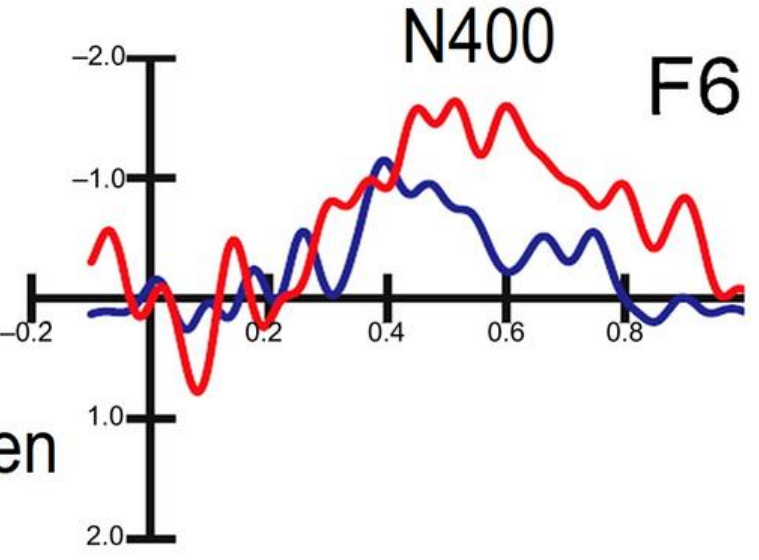

FIGURE 2ERP difference waves obtained by subtracting ERPs to congruent from ERPs to incongruent actions separately for men and women, over anterior scalp sites. It can be appreciated a much larger N400 response to incongruent actions in women than men (original figure, relative to Proverbio et al., 2010c, courtesy of the authors)

A conjoined fMRI and ERP study from our group (Canessa et al., 2012; Proverbio, Riva, Paganelli, et al., 2011) reported a greater female activation of the so-called action understanding system, the ventral premotor cortex and the superior temporal sulcus (STS) in a task involving the observation of social interactions with a cooperative or an affective goal. Other studies provided several lines of evidence of sex differences in the brain development of perceptual systems devoted to biological motion. fMRI data collected during visual perception of coherent versus incoherent biological motion have found enhanced activity in females versus males especially focused in areas devoted to social perception (such as, the temporal pole and the medial temporal gyrus).

\subsection{Face pareidolia}

Due to the social bias, females would also be more inclined than men at seeing faces in things (face pareidolia), as recently shown by behavioral and electrophysiological findings (Pavlova et al., 2015; Proverbio \& Galli, 2016; Zhou \& Meng, 2020). Pavlova et al. (2015) compared women and men in a face recognition task involving Arcimboldo-like food patterns and found that women were more inclined to recognize a face in the food configuration and gave food displays higher face-likeness ratings. Interestingly, Proverbio and Galli (2016) further investigated this issue by recording EEG/ERP in women and men engaged in viewing photographs of animals (targets), 
objects, faces in things, and faces. Overall, compared to men, women were more inclined to see faces in perfectly real objects, as shown in the preliminary face-likeness ratings assessment.

Furthermore, vertex positive potential of the ERPs showed a discriminative response between faces and faces-in things in men (with larger responses to faces), while it was of comparable amplitude in women, clearly reflecting a face pareidolia effect. The analysis of intracranial generator of this response in women identified the orbitofrontal cortex, the right STS and the posterior cingulate cortex as the stronger sources of activity for the affective processing (face likeness) of things. According to the authors, this network would support the anthropomorphizing bias in females.

\subsection{Empathy for pain}

Recent findings have demonstrated that women might be more reactive than men at the view of suffering conspecifics (vicarious response to pain), and therefore more empathic (Han et al., 2008). This sex difference fully agrees with Baron-Cohen model, as well as with a recent survey examining the empathy quotients of 671,606 individuals (Greenberg et al., 2018). In a study by Proverbio et al. (2009) it was investigated whether the two sexes differed in their cerebral response to affective pictures, depicting people in different positive or negative contexts compared to natural or urban landscapes. More than 400 stimuli from the International Affective Picture System (IAPS) were shown to a group of female and male students. An emotional impact scale was administered to all participants showing higher emotional psychological reactions in women than men to a variety of emotional stimuli.

Visual P1 response was larger in amplitude to persons than to landscapes and was modulated by the emotional valence of the social information. A late positive response to negatively valence social information (suffering humans, visible in Figure 3, blue line) far exceeded the response to negative nonsocial information in females but not in males. Again, a low-resolution electromagnetic tomography applied to ERP data showed an enhanced activation of the right amygdala and of the right frontal areas in women (compared to men) for the contrast suffering-minus-happy humans. This functional difference possibly suggests a stronger empathic response in the female brain at the sight of the others' pain. 


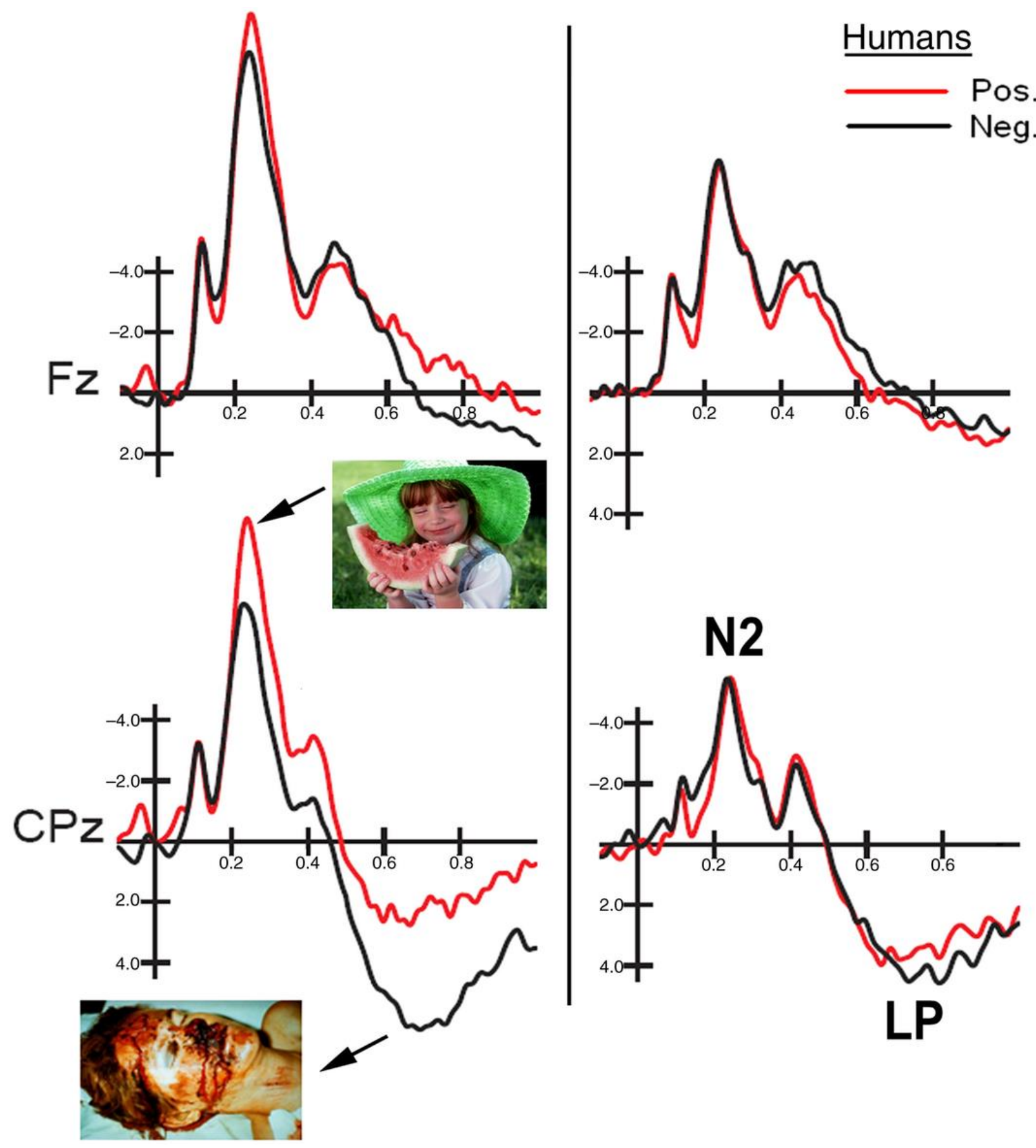

FIGURE 3ERPs recorded at midline frontal and centro/parietal sites as a function of stimulus valence and viewer's sex. It is visible a large effect of both emotional content (that can be appreciated by comparing ERPs to negative vs. positive unanimated scenes), and an effect of empathy for pain, especially in women (that can be appreciated by comparing ERPs to negative scenes vs. ERPs to pictures portraying humans). Original figure, relative to Proverbio et al. (2009)'s study, reproduced with permission of the authors and of Elsevier

Hofer et al. (2007) found larger activation of the right superior temporal area, right insula, right putamen, and ACC during processing of affective words for women versus men, and interpreted this finding as reflecting the greater emotionality of female individuals. Again, Klein et al. (2003) found increased activation of the anterior cingulate cortex and the amygdala in women in response to negative IAPS pictures. In the study by Proverbio and coauthors (2009), sex differences in the response to negative versus positive stimuli were much greater for social than nonsocial information. This might suggest that visual images of humans have a special status for the female 
brain, especially in interaction with negative valence. These data are consistent with other literature suggesting the women might be more empathic than men at the view of suffering humans (Han et al., 2008; Schulte-Rüther et al., 2008; Singer et al., 2004).

In summary, we have here outlined a series of sex differences in social behavioral and psychological processes that seem biologically associated with female chromosomal karyotype. They are: efficient and bilateral face processing, marked empathic attitude, sensitivity to facial mimicry and gestures, attachment to infants (induced by oxytocin), early interest for infants, curiosity about people and social information, emotional responsivity, lesser incidence of autistic, psychopathic, and sociopathic disorders. Knowledge of sex differences in the brain is essential for understanding how to treat male and female patients with sex-specific medications (Cahill, 2017), or how to recognize and treat gender-specific symptoms in socially related disorders (e.g., autistic spectrum disorder, attention deficit/hyperactivity disorder, personality disorder and psychopathy, conduct disorder, alexitimia, depression, social anxiety etc., American Psychiatric Association, 2013). The greater incidence of some of these disorders in the male population might ultimately be due to sex differences in social cognition.

\section{ACKNOWLEDGMENTS}

SThis work was supported by13974 2015-ATE-0052 grant entitled "Emotional responses and gender differences in individuals with high traits of psychopathy, impulsivity and empathy" from the University of Milano-Bicocca.

\section{CONFLICT OF INTEREST}

No conflicting interests exist. 